\title{
Contraction Mappings and Fixed Point in 2-Banach Spaces
}

\section{Risto Malčeski}

Faculty of Informatics,

FON University, Skopje, Macedonia risto.malceski@gmail.com

\section{Alit Ibrahimi}

University of Tetova,

Macedonia

ibraimia@yahoo.com

\begin{abstract}
In [2] Kir and Kiziltunc, in case of 2-Banach spaces, give generalization of theorem for fixed point of Kannan ([6]) and Chatterjea ([10]). In this paper are proven several theorems, which are generalizations of the results of Kannan and Chatterjea, as well as the results of Koparde and Waghmode ([5]).
\end{abstract}

Keywords: 2-normed spaces, 2-Banach spaces, fixed point, contraction mappings

2010 Mathematics Subject Classification. 46J10, 46J15, 47H10

\section{INTRODUCTION}

R. Kannan ([6]) 1968 proved that if $(X, d)$ is a complete metric space and $S: X \rightarrow X$ is mapping, it exists $\alpha \in\left(0, \frac{1}{2}\right)$ such that for all $x, y \in X$ so that the inequality

$$
d(S x, S y) \leq \alpha(d(x, S x)+d(y, S y))
$$

is satisfied, then $S$ has a unique fixed point. S. K. Chatterjea ([10]) 1972 proved that if $(X, d)$ is a complete metric space and $S: X \rightarrow X$ is mapping, it exists $\alpha \in\left(0, \frac{1}{2}\right)$ such that for all $x, y \in X$ so that the inequality

$$
d(S x, S y) \leq \alpha(d(x, S y)+d(y, S x))
$$

holds true, then $S$ has a unique fixed point.

Gähler ([9]) 1965 gave the term of 2-normed space, and White ([1]) 1968 the term of 2-Banach space. Some results about 2-Banach spaces are given in [7]. In this paper we will firstly give some basic definitions and results in a vector 2-normed space.

Definition 1 ([9]). Let $L$ be a real vector space with $\operatorname{dim} L \geq 2$. 2-norm is a real function $\|\cdot, \cdot\|: L \times L \rightarrow[0, \infty)$ such that:

a) $\|x, y\| \geq 0$, for all $x, y \in L$ and $\|x, y\|=0$ if and only if the set $\{x, y\}$ is linearly dependent,

b) $\|x, y\|=\|y, x\|$, for all $x, y \in L$,

c) $\|\alpha x, y\|=|\alpha| \cdot\|x, y\|$, for all $x, y \in L$ and for each $\alpha \in \mathbf{R}$, and

d) $\|x+y, z\| \leq\|x, z\|+\|y, z\|$, for all $x, y, z \in L$.

The ordered pair $(L,\|\cdot, \cdot\|)$ is called a 2-normed space.

Deffinition 2 ([1]). The sequence $\left\{x_{n}\right\}_{n=1}^{\infty}$ into vector 2-normed space is called as convergent if there exists $x \in L$ such that $\lim _{n \rightarrow \infty}\left\|x_{n}-x, y\right\|=0$, for every $y \in L$. Vector $x \in L$ is called as bound of the sequence $\left\{x_{n}\right\}_{n=1}^{\infty}$ and we note $\lim _{n \rightarrow \infty} x_{n}=x$ or $x_{n} \rightarrow x, n \rightarrow \infty$. 
Deffinition 3 ([3]). Let $L$ be a 2-normed space. The mapping $S: L \rightarrow L$ is said continuous mapping in $x$ if for any sequence $\left\{x_{n}\right\}$ in $L$ such that $x_{n} \rightarrow x$ for $n \rightarrow \infty$ the following holds true $S x_{n} \rightarrow S x$, $n \rightarrow \infty$.

Deffinition 4 ([1]). The sequence $\left\{x_{n}\right\}_{n=1}^{\infty}$ in a vector 2-normed space $L$ is Cauchy sequence if $\lim _{m, n \rightarrow \infty}\left\|x_{n}-x_{m}, y\right\|=0$, for every $y \in L$. For 2-normed space $L$, we'll say that is $n$-Banach space if every Cauchy sequence is convergent.

Deffinition 5 ([4]). Let $(L,\|\cdot, \cdot\|)$ be a real vector 2-normed space and $C$ be a subset of $L$. The set

$$
\bar{C}=\left\{x \mid x \in L \text { and there is a sequence } x_{n} \text { of } C \text { such that } x_{n} \rightarrow x\right\}
$$

is said closure on $C$. If $C=\bar{C}$, then $C$ is said closed in $L$.

Deffinition 6 ([4]). Let $(L,\|\cdot, \cdot\|)$ be a real vector 2-normed space, $B$ be a nonempty subset of $L$ and $e \in B$. The set $B$ is said $e$-bounded if it exists a real positive number $M>0$, so that $\|x, e\| \leq M$, for each $x \in B$. If $B$ is $e$-bounded for each $e \in B$, then $B$ is said bounded set.

Hatikrishnan and Ravindran in [4] gave the term of contractive mapping in 2-normed space as the following:

Deffinition 7 ([4]). Let $(L,\|, \cdot\|)$ be a real vector 2-normed space. The mapping $S: L \rightarrow L$ is contraction if it exists $\lambda \in[0,1)$ so that $\|S x-S y, z\| \leq \lambda\|x-y, z\|$, for all $x, y, z \in L$, holds true.

Hatikrishnan and Ravindran in [4] proved that contractive mapping has a unique fixed point in a closed and bounded subset of 2-Banach space. Further, Kir and Kiziltunc proved that if $C$ is a nonempty and closed subset of 2-Banach space and $S: C \rightarrow C$ is such that it satisfies one of the following conditions

$$
\|S x-S y, z\| \leq \alpha(\|x-S x, z\|+\|y-S y, z\|), \text { for all } x, y, z \in C
$$

or

$$
\|S x-S y, z\| \leq \alpha(\|x-S y, z\|+\|y-S x, z\|), \text { for all } x, y, z \in C
$$

for $\alpha \in\left(0, \frac{1}{2}\right)$, then $S$ has a unique fixed point in $C$ (theorem 1, [2] and theorem 2, [2], respectively). If the condition (1) is satisfied, then we get generalization of Kannan theorem, otherwise if the condition (2) is satisfied, then we get the generalization of Chatterjea theorem.

\section{Generalizations of Kannan and Chatterjea Theorems}

Theorem 1. Let $(L,\|\cdot \cdot\|)$ be a 2-Banach space and $S: L \rightarrow L$ be mapping, so that

$$
\|S x-S y, z\| \leq \alpha(\|x-S x, z\|+\|y-S y, z\|)+\gamma\|x-y, z\|,
$$

for all $x, y, z \in L$, and $\alpha>0, \gamma \geq 0$ and $0<2 \alpha+\gamma<1$. Then $S$ has a unique fixed point in $L$.

Proof. Let $x_{0}$ be any point on $L$ and the sequence $\left\{x_{n}\right\}$ be defined as the following $x_{n+1}=S x_{n}$, $n=0,1,2, \ldots$. The inequality (3) implies that

$$
\begin{gathered}
\left\|x_{n+1}-x_{n}, z\right\|=\left\|S x_{n}-S x_{n-1}, z\right\| \leq \alpha\left(\left\|x_{n}-S x_{n}, z\right\|+\left\|x_{n-1}-S x_{n-1}, z\right\|\right)+\gamma\left\|x_{n}-x_{n-1}, z\right\| \\
=\alpha\left(\left\|x_{n}-x_{n+1}, z\right\|+\left\|x_{n-1}-x_{n}, z\right\|\right)+\gamma\left\|x_{n}-x_{n-1}, z\right\|,
\end{gathered}
$$


holds true for each $n=0,1,2, \ldots$ and each $z \in L$. The condition of the Theorem implies that $\lambda=\frac{\alpha+\gamma}{1-\alpha}<1$. Therefore, the latter implies that

$$
\left\|x_{n+1}-x_{n}, z\right\| \leq \lambda\left\|x_{n}-x_{n-1}, z\right\|
$$

holds true for each non-negative $n=0,1,2, \ldots$ and each $z \in L$. Further, (4) implies that

$$
\left\|x_{n+1}-x_{n}, z\right\| \leq \lambda^{n}\left\|x_{1}-x_{0}, z\right\|,
$$

holds true for each $n=0,1,2, \ldots$ and each $z \in L$. Using the properties of 2-norm and (5) we get that

$$
\begin{gathered}
\left\|x_{m+k}-x_{k}, z\right\| \leq\left\|x_{m+k}-x_{m+k-1}, z\right\|+\left\|x_{m+k-1}-x_{m+k-2}, z\right\|+\ldots+\left\|x_{k+1}-x_{k}, z\right\| \\
\leq\left(\lambda^{m+k-1}+\lambda^{m+k-2}+\ldots+\lambda^{k}\right)\left\|x_{1}-x_{0}, z\right\| \leq \frac{\lambda^{k}}{1-\lambda}\left\|x_{1}-x_{0}, z\right\|
\end{gathered}
$$

holds true for all $m, k \in \mathbf{N}$ and each $z \in L$. Since $\lambda<1$, the latter implies that

$$
\lim _{m, k \rightarrow \infty}\left\|x_{k}-x_{k+m}, z\right\|=0
$$

holds true for each $m \in \mathbf{N}$ and each $z \in L$. The latter actually means that the sequence $\left\{x_{n}\right\}$ is Caushy sequence. But the space $L$ is 2-Banach, therefore the sequence $\left\{x_{n}\right\}$ is convergent sequence, i.e. it exists $u \in L$ so that $\lim _{n \rightarrow \infty} x_{n}=u$. We will prove that $u$ is a fixed point on $S$. Thus,

$$
\begin{aligned}
\|u-S u, z\| & \leq\left\|u-x_{n+1}, z\right\|+\left\|x_{n+1}-S u, z\right\|=\left\|u-x_{n+1}, z\right\|+\left\|S x_{n}-S u, z\right\| \\
& \leq\left\|u-x_{n+1}, z\right\|+\alpha\left(\left\|x_{n}-S x_{n}, z\right\|+\|u-S u, z\|\right)+\gamma\left\|u-x_{n}, z\right\| \\
& =\left\|u-x_{n+1}, z\right\|+\alpha\left(\left\|x_{n}-x_{n+1}, z\right\|+\|u-S u, z\|\right)+\gamma\left\|u-x_{n}, z\right\|,
\end{aligned}
$$

for each $n \in \mathbf{N}$ and each $z \in L$. For $n \rightarrow \infty$, the latter is transformed as the following $\|u-S u, z\| \leq \alpha\|u-S u, z\|$, for each $z \in L$. Since, $\alpha<1$ it is true that $\|u-S u, z\|=0$, for each $z \in L$. Therefore, $S u=u$, i.e. $u$ is a fixed point on $S$. We will prove that $S$ has a unique fixed point. Let $u, v \in L$ be fixed point on $S$, i.e. $S u=u$ and $S v=v$. So,

$$
\|u-v, z\|=\|S u-S v, z\| \leq \alpha(\|u-S u, z\|+\|v-S v, z\|)+\gamma\|u-v, z\|=\gamma\|u-v, z\|,
$$

holds true, for each $z \in L$. Since $\gamma<1$, the latter implies that $\|u-v, z\|=0$, for each $z \in L$, therefore $u=v$.

Remark 1. For $\gamma=0$, the condition (3) transforms as (1). That actually means that Theorem 1, [2] is Consequence of the Theorem 1.

Consequence 1. Let $(L,\|, \cdot\|)$ be a 2-Banach space and $S: L \rightarrow L$ be mapping so that

$$
\|S x-S y, z\| \leq \lambda \cdot \sqrt[3]{\|x-S x, z\| \cdot\|y-S y, z\| \cdot\|x-y, z\|}
$$

holds true for all $x, y, z \in L$, and $\lambda \in(0,1)$. Then $S$ has a unique fixed point in $L$.

Proof. The inequality stated in the condition and the arithmetic-geometric mean inequality imply that

$$
\|S x-S y, z\| \leq \lambda \cdot \sqrt[3]{\|x-S x, z\| \cdot\|y-S y, z\| \cdot\|x-y, z\|} \leq \lambda \cdot \frac{\|x-S x, z\|+\|y-S y, z\|+\|x-y, z\|}{3} .
$$

Finally, the statement is implied by Theorem 1 for $\alpha=\gamma=\frac{\lambda}{3}$. 
Consequence 2. Let $(L,\|\cdot \cdot\|)$ be a 2-Banach space and $S: L \rightarrow L$ be mapping so that

$$
\|S x-S y, z\| \leq \alpha \frac{\|x-S x, z\|^{2}+\|y-S y, z\|^{2}}{\|x-S x, z\|+\|y-S y, z\|}+\gamma\|x-y, z\|
$$

holds true for all $x, y, z \in L, z \neq 0$, and $\alpha>0, \gamma \geq 0,0<2 \alpha+\gamma<1$. Then $S$ has a unique fixed point in $L$.

Proof. The inequality given in the condition implies that (1) holds true for all $x, y, z \in L$. The statement is directly implied by Theorem 1 .

Theorem 2. Let $(L,\|\cdot, \cdot\|)$ be a 2-Banach space and $S: L \rightarrow L$ be mapping so that

$$
\|S x-S y, z\| \leq \alpha(\|x-S y, z\|+\|y-S x, z\|)+\gamma\|x-y, z\|,
$$

holds true for all $x, y, z \in L$, and $\alpha>0, \gamma \geq 0,2 \alpha+\gamma<1$. Then $S$ has a unique fixed point in $L$.

Proof. Let $x_{0}$ be any point on $L$ and the sequence $\left\{x_{n}\right\}$ be defined as the following $x_{n+1}=S x_{n}$, $n=0,1,2,3, \ldots$. The inequality (6) implies validity of the following

$$
\begin{aligned}
\left\|x_{n+1}-x_{n}, z\right\| & =\left\|S x_{n}-S x_{n-1}, z\right\| \leq \alpha\left(\left\|x_{n}-S x_{n-1}, z\right\|+\left\|x_{n-1}-S x_{n}, z\right\|\right)+\gamma\left\|x_{n}-x_{n-1}, z\right\| \\
& =\alpha\left\|x_{n-1}-x_{n+1}, z\right\|+\gamma\left\|x_{n}-x_{n-1}, z\right\| \\
& \leq \alpha\left\|x_{n-1}-x_{n}, z\right\|+\alpha\left\|x_{n}-x_{n+1}, z\right\|+\gamma\left\|x_{n}-x_{n-1}, z\right\|
\end{aligned}
$$

for each $n=0,1,2, \ldots$ and each $z \in L$, i.e.

$$
\left\|x_{n}-x_{n+1}, z\right\| \leq \lambda\left\|x_{n}-x_{n-1}, z\right\|
$$

for each $n=0,1,2, \ldots$ and each $z \in L$, and $\lambda=\frac{\alpha+\gamma}{1-\alpha}<1$. Further, (7) implies that

$$
\left\|x_{n+1}-x_{n}, z\right\| \leq \lambda^{n}\left\|x_{1}-x_{0}, z\right\|
$$

holds true for each $n=0,1,2, \ldots$ and each $z \in L$. Analogously, as the proof of the Theorem 1, the sequence $\left\{x_{n}\right\}$ is convergent, i.e. it exists $u \in L$ so that $\lim _{n \rightarrow \infty} x_{n}=u$. We will prove that $u$ is a fixed point on $S$. Thus,

$$
\begin{array}{r}
\|u-T u, z\| \leq\left\|u-x_{n+1}, z\right\|+\left\|x_{n+1}-S u, z\right\|=\left\|u-x_{n+1}, z\right\|+\left\|S x_{n}-S u, z\right\| \\
\quad \leq\left\|u-x_{n+1}, z\right\|+\alpha\left(\left\|x_{n}-S u, z\right\|+\left\|u-S x_{n}, z\right\|\right)+\gamma\left\|u-x_{n}, z\right\| \\
\quad=\left\|u-x_{n+1}, z\right\|+\alpha\left(\left\|x_{n}-S u, z\right\|+\left\|u-x_{n+1}, z\right\|\right)+\gamma\left\|u-x_{n}, z\right\|
\end{array}
$$

holds true for each $n \in \mathbf{N}$ and each $z \in L$. For $n \rightarrow \infty$ the last inequality is transformed as the following $\|u-S u, z\| \leq \alpha\|u-S u, z\|$, for each $z \in L$. Since $\alpha<1$, we get that $\|u-S u, z\|=0$, for each $z \in L$. Therefore, $S u=u$, i.e. $u$ is a fixed point on $S$. We will prove that $S$ has a unique fixed point. Let $u, v \in L$ be two different fixed points on $S$, i.e. $S u=u$ and $S v=v$. Thus,

$$
\|u-v, z\|=\|S u-S v, z\| \leq \alpha(\|u-S v, z\|+\|v-S u, z\|)+\gamma\|u-v, z\|=(2 \alpha+\gamma)\|u-v, z\|,
$$

holds true for each $z \in L$. Since $2 \alpha+\gamma<1$, the last inequality implies that $\|u-v, z\|=0$, for each $z \in L$. Therefore, $u=v$.

Remark 2. For $\gamma=0$, the condition (7) is transformed as (2). That is the theorem 2, [2] is consequence of the theorem 2 . 
Consequence 3. Let $(L,\|, \cdot\|)$ be a 2-Banach space and $S: L \rightarrow L$ be a mapping so that

$$
\|S x-S y, z\| \leq \lambda \cdot \sqrt[3]{\|x-S y, z\| \cdot\|y-S x, z\| \cdot\|x-y, z\|},
$$

for all $x, y, z \in L$, and $\lambda \in(0,1)$ holds true. Then, $S$ has a unique fixed point in $L$.

Proof. The inequality stated in the condition and the arithmetic-geometric mean inequality imply that

$$
\|S x-S y, z\| \leq \lambda \cdot \sqrt[3]{\|x-S y, z\| \cdot\|y-S x, z\| \cdot\|x-y, z\|} \leq \lambda \cdot \frac{\|x-S y, z\|+\|y-S x, z\|+\|x-y, z\|}{3} .
$$

holds true. The statement is a direct implication by the theorem 2 for $\alpha=\gamma=\frac{\lambda}{3}$.

Consequence 4. Let $(L,\|, \cdot\|)$ be a 2-Banach space and $S: L \rightarrow L$ be a mapping so that

$$
\|S x-S y, z\| \leq \alpha \frac{\|x-S y, z\|^{2}+\|y-S x, z\|^{2}}{\|x-S y, z\|+\|y-S x, z\|}+\gamma\|x-y, z\|,
$$

for all $x, y, z \in L, z \neq 0, \alpha>0, \gamma \geq 0$ and $2 \alpha+\gamma<1$ Then, $S$ has a unique fixed point in $L$.

Proof. The condition inequality implies that (2) holds true for all $x, y, z \in L$. Thus, the statement is a direct implication of the theorem 2.

Koparde and Waghmode in [5] proved that, if $H$ is a Hilbert space, $C$ is closed subset of $H$ and the mapping $S: C \rightarrow C$ is such that

$$
\|S x-S y\|^{2} \leq \alpha\left(\|x-S x\|^{2}+\|y-S y\|^{2}\right),
$$

holds true for all $x, y \in C$, and $\alpha \in\left(0, \frac{1}{2}\right)$, then $S$ has a unique fixed point in $C$. In the following consideration we will generalize the above result in 2-Banach spaces.

Theorem 3. Let $(L,\|\cdot \cdot\|)$ be a 2-Banach space and $S: L \rightarrow L$ be a mapping such that

$$
\|S x-S y, z\|^{2} \leq \alpha\left(\|x-S x, z\|^{2}+\|y-S y, z\|^{2}\right)+\gamma\|x-y, z\|^{2},
$$

holds true for all $x, y, z \in L$, and $\alpha>0, \gamma \geq 0,2 \alpha+\gamma<1$. Then $S$ has a unique fixed point in $L$.

Proof. Let $x_{0}$ be any point in $L$ and the sequence $\left\{x_{n}\right\}$ be defined as the following $x_{n+1}=S x_{n}$, $n=0,1,2,3, \ldots$. The inequality (12) implies that

$$
\begin{aligned}
\left\|x_{n+1}-x_{n}, z\right\|^{2} & =\left\|S x_{n}-S x_{n-1}, z\right\|^{2} \leq \alpha\left(\left\|x_{n}-S x_{n}, z\right\|^{2}+\left\|x_{n-1}-S x_{n-1}, z\right\|^{2}\right)+\gamma\left\|x_{n}-x_{n-1}, z\right\|^{2} \\
& =\alpha\left(\left\|x_{n}-x_{n+1}, z\right\|^{2}+\left\|x_{n-1}-x_{n}, z\right\|^{2}\right)+\gamma\left\|x_{n}-x_{n-1}, z\right\|^{2},
\end{aligned}
$$

holds true for each $n=0,1,2, \ldots$ and each $z \in L$. The condition stated in the above theorem implies that $\lambda=\sqrt{\frac{\alpha+\gamma}{1-\alpha}}<1$. Therefore, the latter inequality implies that

$$
\left\|x_{n+1}-x_{n}, z\right\| \leq \lambda\left\|x_{n}-x_{n-1}, z\right\|
$$

holds true for each $n=0,1,2, \ldots$ and each $z \in L$. Thus, the inequality (13) implies that

$$
\left\|x_{n+1}-x_{n}, z\right\| \leq \lambda^{n}\left\|x_{1}-x_{0}, z\right\|
$$


for each $n=0,1,2, \ldots$ and each $z \in L$, holds true. Further, analogously as the proof of the Theorem 1 , the sequence $\left\{x_{n}\right\}$ is convergent, i.e. it exists $u \in L$ so that $\lim _{n \rightarrow \infty} x_{n}=u$. We will prove that $u$ is a fixed point of $S$. Thus,

$$
\begin{aligned}
\|u-S u, z\| \leq & \left\|u-x_{n+1}, z\right\|+\left\|x_{n+1}-S u, z\right\|=\left\|u-x_{n+1}, z\right\|+\left\|S x_{n}-S u, z\right\| \\
& \leq\left\|u-x_{n+1}, z\right\|+\sqrt{\alpha\left(\left\|x_{n}-S x_{n}, z\right\|^{2}+\|u-S u, z\|^{2}\right)+\gamma\left\|u-x_{n}, z\right\|^{2}} \\
& =\left\|u-x_{n+1}, z\right\|+\sqrt{\alpha\left(\left\|x_{n}-x_{n+1}, z\right\|^{2}+\|u-S u, z\|^{2}\right)+\gamma\left\|u-x_{n}, z\right\|^{2}},
\end{aligned}
$$

holds true for each $n \in \mathbf{N}$ and each $z \in L$. For $n \rightarrow \infty$ the latter is transformed as the following $\|u-S u, z\| \leq \sqrt{\alpha}\|u-S u, z\|$, for each $z \in L$. Further, $\sqrt{\alpha}<1$, and the last inequality implies that $\|u-S u, z\|=0$, for each $z \in L$. The latter implies that $S u=u$, i.e. $u$ is a fixed point of $S$. We will prove that $S$ has a unique fixed point. Let $u, v \in L$ be two different fixed point of $S$, i.e. $S u=u$ and $S v=v$. Thus,

$$
\|u-v, z\|^{2}=\|S u-S v, z\|^{2} \leq \alpha\left(\|u-S u, z\|^{2}+\|v-S v, z\|^{2}\right)+\gamma\|u-v, z\|^{2}=\gamma\|u-v, z\|^{2},
$$

for each $z \in L$. Since $\sqrt{\gamma}<1$ the latter implies $\|u-v, z\|=0$, for each $z \in L$. Therefore $u=v$.

Consequence 5. Let $(L,\|, \cdot\|)$ be a 2-Banach space and $S: L \rightarrow L$ be a mapping so that

$$
\|S x-S y, z\|^{2} \leq \alpha\left(\|x-S x, z\|^{2}+\|y-S y, z\|^{2}\right),
$$

holds true, for all $x, y, z \in L$ and $\alpha \in\left(0, \frac{1}{2}\right)$. Then $T$ has a unique fixed point in $L$.

Proof. For $\gamma=0$ in the Theorem 3, we get the required statement.

\section{Common Fixed Points of Mappings}

Theorem 4. Let $(L,\|\cdot \cdot\|)$ be a 2-Banach space and $S_{1}, S_{2}: L \rightarrow L$ be mappings so that

$$
\left\|S_{1} x-S_{2} y, z\right\| \leq \alpha\left(\left\|x-S_{1} x, z\right\|+\left\|y-S_{2} y, z\right\|\right)+\beta\|x-y, z\|,
$$

holds true for all $x, y, z \in L$, and $\alpha>0, \beta \geq 0,2 \alpha+\beta<1$. Then $S_{1}$ and $S_{2}$ have a common fixed point $z \in L$.

Proof. Let $x_{0}$ be any point in $L$. We define a sequence $\left\{x_{n}\right\}$ in $L$ so that $x_{2 n+1}=S_{1} x_{2 n}$, $x_{2 n+2}=S_{2} x_{2 n+1}$, holds true, for $n=0,1,2, \ldots$. If it exists $n \geq 0$ so that $x_{n}=x_{n+1}=x_{n+2}$ is satisfied, then it is easy to be proven that $z=x_{n} \in L$ is a common fixed point in $S_{1}$ and $S_{2}$. Therefore, let suppose that it does not exist three equivalent consecutive terms of the sequence $\left\{x_{n}\right\}$. Thus,

$$
\begin{aligned}
\left\|x_{2 n+1}-x_{2 n}, z\right\| & =\left\|S_{1} x_{2 n}-S_{2} x_{2 n-1}, z\right\| \\
& \leq \alpha\left(\left\|x_{2 n}-S_{1} x_{2 n}, z\right\|+\left\|x_{2 n-1}-S_{2} x_{2 n-1}, z\right\|\right)+\beta\left\|x_{2 n}-x_{2 n-1}, z\right\| \\
& \leq \alpha\left(\left\|x_{2 n+1}-x_{2 n}, z\right\|+\left\|x_{2 n}-x_{2 n-1}, z\right\|\right)+\beta\left\|x_{2 n}-x_{2 n-1}, z\right\|,
\end{aligned}
$$

holds true for each $n \geq 1$ and each $z \in L \backslash\{0\}$. Analogously, can be proven validity of the following inequality

$$
\left\|x_{2 n-1}-x_{2 n}, z\right\| \leq \alpha\left(\left\|x_{2 n-2}-x_{2 n-1}, z\right\|+\left\|x_{2 n}-x_{2 n-1}, z\right\|\right)+\beta\left\|x_{2 n-2}-x_{2 n-1}, z\right\| .
$$

The latter implies that 


$$
\left\|x_{n+1}-x_{n}, z\right\| \leq \lambda\left\|x_{n}-x_{n-1}, z\right\|
$$

holds true for each $n \geq 1$ and each $z \in L$, for $\lambda=\frac{\alpha+\beta}{1-\alpha}<1$. Further, the inequality (14), analogously as in the Theorem 1, implies that the sequence $\left\{x_{n}\right\}$ is a Caushy sequence, that is, $\left\{x_{n}\right\}$ is a convergent sequence, i.e. it exists $u \in L$ so that $\lim _{n \rightarrow \infty} x_{n}=u$. We will prove that $u$ is a fixed point of $S_{1}$. So,

$$
\begin{aligned}
& \left\|u-S_{1} u, z\right\| \leq\left\|u-x_{2 n+2}, z\right\|+\left\|x_{2 n+2}-S_{1} u, z\right\|=\left\|u-x_{2 n+2}, z\right\|+\left\|S_{2} x_{2 n+1}-S_{1} u, z\right\| \\
& \quad \leq\left\|u-x_{2 n+2}, z\right\|+\alpha\left(\left\|x_{2 n+1}-S_{2} x_{2 n+1}, z\right\|+\left\|u-S_{1} u, z\right\|\right)+\beta\left\|u-x_{2 n+1}, z\right\| \\
& \quad \leq\left\|u-x_{2 n+2}, z\right\|+\alpha\left(\left\|x_{2 n+1}-x_{2 n+2}, z\right\|+\left\|u-S_{1} u, z\right\|\right)+\beta\left\|u-x_{2 n+1}, z\right\|
\end{aligned}
$$

holds true for each $n \geq 1$ and each $z \in L \backslash\{0\}$. For $n \rightarrow \infty$, the latter inequality is transformed as the following $\left\|u-S_{1} u, z\right\| \leq \alpha\left\|u-S_{1} u, z\right\|$, for each $z \in L$. But, $\alpha<1$, and therefore the latter implies that $\left\|u-S_{1} u, z\right\|=0$, holds true for each $z \in L$. Thus, $S_{1} u=u$, i.e. $u$ is a fixed point of $S_{1}$. Analogously, can be proven that $u$ is a fixed point of $S_{2}$, that is, $u$ is a fixed point of $S_{1}$ and $S_{2}$. We will prove that $S_{1}$ and $S_{2}$ have a unique common fixed point. Let $v \in L$ be one other fixed point of $S_{2}$, i.e. $S_{2} v=v$. Thus,

$$
\|u-v, z\|=\left\|S_{1} u-S_{2} v, z\right\| \leq \alpha\left(\left\|u-S_{1} u, z\right\|+\left\|v-S_{2} v, z\right\|\right)+\beta\|u-v, z\|=\beta\|u-v, z\|,
$$

holds true. Since $\beta<1$, we get that $\|u-v, z\|=0$. Therefore $u=v$.

Consequence 6. Let $(L,\|\cdot\|$,$) be a 2-Banach space and S_{1}, S_{2}: L \rightarrow L$ be mappings, so that

$$
\left\|S_{1} x-S_{2} y, z\right\| \leq \alpha \frac{\left\|x-S_{1} x, z\right\|^{2}+\left\|y-S_{2} y, z\right\|^{2}}{\left\|x-S_{1} x, z\right\|+\left\|y-S_{2} y, z\right\|}+\beta\|x-y, z\|,
$$

holds true for all $x, y, z \in L, z \neq 0$, and $\alpha>0, \beta \geq 0,2 \alpha+\beta<1$. Then $S_{1}$ and $S_{2}$ have a common fixed point $z \in L$.

Proof. The inequality stated in the condition, for $z \neq 0$, directly implies the inequality (13). For $z=0$, the inequality (13) obviously holds true. And finally, the statement is directly implied by the Theorem 4.

Consequence 7. Let $(L,\|\cdot, \cdot\|)$ be a 2-Banach space and $S_{1}, S_{2}: L \rightarrow L$ be mappings so that

$$
\left\|S_{1} x-S_{2} y, z\right\| \leq \lambda \cdot \sqrt[3]{\left\|x-S_{1} x, z\right\| \cdot\left\|y-S_{2} y, z\right\| \cdot\|x-y, z\|},
$$

holds true for all $x, y, z \in L$, and $\lambda \in(0,1)$. Then $S$ has a unique fixed point in $L$.

Proof. The arithmetic-geometric mean inequality and the Theorem 4 , for $\alpha=\beta=\frac{\lambda}{3}$, directly imply the statement given in the Consequence 7.

Consequence 8. Let $(L,\|\cdot, \cdot\|)$ be a 2-Banach space and $p, q \in \mathbf{N}$ be such that there exists mappings $S_{1}^{p}, S_{2}^{q}: L \rightarrow L$ and also exists $\alpha>0, \beta \geq 0,2 \alpha+\beta<1$ such that they satisfy the following

$$
\left\|S_{1}^{p} x-S_{2}^{q} y, z\right\| \leq \alpha\left(\left\|x-S_{1}^{p} x, z\right\|+\left\|y-S_{2}^{q} y, z\right\|\right)+\beta\|x-y, z\|,
$$

for all $x, y, z \in L$. Then $S_{1}$ and $S_{2}$ have a unique common fixed point $u \in L$. 
Proof. According to the Theorem 4, the mappings $S_{1}^{p}$ and $S_{2}^{q}$ have a unique common fixed point $u \in L$. Thus, $S_{1}^{p} u=u$. So, $S_{1} u=S_{1}\left(S_{1}^{p} u\right)=S_{1}^{p}\left(S_{1} u\right)$, that is, $S_{1} u$ is a fixed point of $S_{1}^{p}$. Analogously, $S_{2}^{q} u=u$ implies $S_{2} u=S_{2}\left(S_{2}^{q} u\right)=S_{2}^{q}\left(T_{2} u\right)$, that is, $S_{2} u$ is a fixed point of $S_{2}^{q}$. But, both $S_{2}^{q}$ and $S_{1}^{p}$ have a unique fixed point. Therefore, $u=S_{2} u$ and $u=S_{1} u$. Thus, $u \in L$ is a common fixed point of $S_{1}$ and $S_{2}$.

If, $v \in L$ is one other fixed point of $S_{1}$ and $S_{2}$, then it is also, a common fixed point of $S_{1}^{p}$ and $S_{2}^{q}$. But, since $S_{1}^{p}$ and $S_{2}^{q}$ have a unique common fixed point, it is true that $u=v$.

Theorem 5. Let $(L,\|\cdot, \cdot\|)$ be a 2-Banach space and $S_{1}, S_{2}: L \rightarrow L$ be mappings so that

$$
\left\|S_{1} x-S_{2} y, z\right\| \leq \alpha\left(\left\|x-S_{2} y, z\right\|+\left\|y-S_{1} x, z\right\|\right)+\beta\|x-y, z\|,
$$

holds true, for all $x, y, z \in L$, and $\alpha>0, \beta \geq 0,2 \alpha+\beta<1$. Then $S_{1}$ and $S_{2}$ have a unique common fixed point $u \in L$.

Proof. Let $x_{0}$ be any point in $L$. Let $\left\{x_{n}\right\}$ be a sequence in $L$, so that $x_{2 n+1}=S_{1} x_{2 n}$, $x_{2 n+2}=S_{2} x_{2 n+1}$, for $n=0,1,2, \ldots$, holds true. If it exists $n \geq 0$, so that $x_{n}=x_{n+1}=x_{n+2}$ is satisfied, then it is easy to be proven that $u=x_{n} \in L$ is a common fixed point of $S_{1}$ and $S_{2}$. Therefore, let's suppose there no exist three equivalent consecutive terms of the sequence $\left\{x_{n}\right\}$. Further, using the inequality (15), analogously as the proof in Theorem 4 it can be proven that

$$
\left\|x_{n+1}-x_{n}, z\right\| \leq \lambda\left\|x_{n}-x_{n-1}, z\right\|
$$

holds true for each $n \geq 1$ and each $z \in L$, for $\lambda=\frac{\alpha+\beta}{1-\alpha}<1$. Analogously as the proof of the Theorem 1 , the inequality (16) implies that the sequence $\left\{x_{n}\right\}$ is convergent, i.e. it exists $u \in L$ so that $\lim _{n \rightarrow \infty} x_{n}=u$. We will prove that $u$ is a fixed point of $S_{1}$. Thus,

$$
\begin{aligned}
\left\|u-S_{1} u, z\right\| & \leq\left\|u-x_{2 n+2}, z\right\|+\left\|x_{2 n+2}-S_{1} u, z\right\|=\left\|u-x_{2 n+2}, z\right\|+\left\|S_{2} x_{2 n+1}-S_{1} u, z\right\| \\
& \quad\left\|u-x_{2 n+2}, z\right\|+\alpha\left(\left\|x_{2 n+1}-S_{1} u, z\right\|+\left\|u-S_{2} x_{2 n+1}, z\right\|\right)+\beta\left\|u-x_{2 n+1}, z\right\| \\
& \leq\left\|u-x_{2 n+2}, z\right\|+\alpha\left(\left\|x_{2 n+1}-S_{1} u, z\right\|+\left\|u-x_{2 n+2}, z\right\|\right)+\beta\left\|u-x_{2 n+1}, z\right\|
\end{aligned}
$$

holds true for each $n \geq 1$ and each $z \in L$. For $n \rightarrow \infty$, the latter is transformed as following $(1-\alpha)\left\|u-S_{1} u, z\right\| \leq 0$, for each $z \in L$. Thereby $1-\alpha>0$, it is true that $\left\|u-S_{1} u, z\right\|=0$, for each $z \in L$. Thus, $S_{1} u=u$, i.e. $u$ is a fixed point of $S_{1}$. Analogously, can be proven that $u$ is a fixed point of $S_{2}$, that is, $u$ is a common fixed point of $S_{1}$ and $S_{2}$. We will prove that $S_{1}$ and $S_{2}$ have a unique common fixed point. Let $v \in L$ be one other fixed point of $S_{2}$, i.e. $S_{2} v=v$. Thus,

$$
\|u-v, z\|=\left\|S_{1} u-S_{2} v, z\right\| \leq \alpha\left(\left\|u-S_{2} v, z\right\|+\left\|v-S_{1} u, z\right\|\right)+\beta\|u-v, z\|=(2 \alpha+\beta)\|u-v, z\|,
$$

holds true. Since $2 \alpha+\beta<1$ it is true that $\|u-v, z\|=0$. The latter implies that $u=v$.

Consequence 9. Let $(L,\|\cdot \cdot\|)$ be a 2-Banach space and $S_{1}, S_{2}: L \rightarrow L$ be mappings, so that

$$
\left\|S_{1} x-S_{2} y, z\right\| \leq \alpha \frac{\left\|x-S_{2} y, z\right\|^{2}+\left\|y-S_{1} x, z\right\|^{2}}{\left\|x-S_{2} y, z\right\|+\left\|y-S_{1} x, z\right\|}+\beta\|x-y, z\|,
$$


holds true for all $x, y, z \in L, z \neq 0$, and $\alpha>0, \beta \geq 0,2 \alpha+\beta<1$. Then $S_{1}$ and $S_{2}$ have a common fixed point $z \in L$.

Proof. For $z \neq 0$, the inequality stated in a condition, directly implies (15). For $z=0$, the inequality (15) also holds true. Finally, the Theorem 5 directly implies the statement.

Consequence 10. Let $(L,\|\cdot, \cdot\|)$ be a 2-Banach space and $S_{1}, S_{2}: L \rightarrow L$ mappings so that

$$
\left\|S_{1} x-S_{2} y, z\right\| \leq \lambda \cdot \sqrt[3]{\left\|x-S_{2} y, z\right\| \cdot\left\|y-S_{1} x, z\right\| \cdot\|x-y, z\|},
$$

is satisfied for all $x, y, z \in L$, for $\lambda \in(0,1)$. Then, $S$ has a unique fixed point in $L$.

Proof. The arithmetic-geometric mean inequality and the Theorem 5, for $\alpha=\beta=\frac{\lambda}{3}$, directly imply the above statement.

Consequence 11. Let $(L,\|\cdot, \cdot\|)$ be a 2-Banach space and $p, q \in \mathbf{N}$ be such that there exists mappings $S_{1}^{p}, S_{2}^{q}: L \rightarrow L$ and also exists $\alpha>0, \beta \geq 0,2 \alpha+\beta<1$ such that they satisfy the following

$$
\left\|S_{1}^{p} x-S_{2}^{q} y, z\right\| \leq \alpha\left(\left\|x-S_{2}^{q} y, z\right\|+\left\|y-S_{1}^{p} x, z\right\|\right)+\beta\|x-y, z\|,
$$

for all $x, y, z \in L$. Then $S_{1}$ and $S_{2}$ have a unique common fixed point $u \in L$.

Proof. The proof is identical to the Proof of Consequence 8 .

Theorem 6. Let $(L,\|\cdot, \cdot\|)$ be a 2-Banach space and $S_{1}, S_{2}: L \rightarrow L$ be mappings, so that they satisfy the following

$$
\left\|S_{1} x-S_{2} y, z\right\|^{2} \leq \alpha\left(\left\|x-S_{1} x, z\right\|^{2}+\left\|y-S_{2} y, z\right\|^{2}\right)+\beta\|x-y, z\|^{2},
$$

for all $x, y, z \in L$, and $\alpha>0, \beta \geq 0,2 \alpha+\beta<1$. Then, $S$ has a unique fixed point in $L$.

Proof. Let $x_{0}$ be any point in $L$. We define a sequence $\left\{x_{n}\right\}$ in $L$ so that $x_{2 n+1}=S_{1} x_{2 n}$, $x_{2 n+2}=S_{2} x_{2 n+1}$, for $n=0,1,2, \ldots$ is satisfied. If it exists $n \geq 0$, so that $x_{n}=x_{n+1}=x_{n+2}$ holds true, then it is easy to be proven that $u=x_{n} \in L$ is a common fixed point of $S_{1}$ and $S_{2}$. Therefore, let's suppose that there no exist three consecutive equivalent terms of the sequence $\left\{x_{n}\right\}$. Further, using the inequality (17), analogously as in the proof of the Theorem 4 it can be proven that

$$
\left\|x_{n+1}-x_{n}, z\right\| \leq \lambda\left\|x_{n}-x_{n-1}, z\right\|,
$$

holds true for each $n=0,1,2, \ldots$ and each $z \in L$, for $\lambda=\sqrt{\frac{\alpha+\beta}{1-\alpha}}<1$. Further, the inequality (18), analogously as in the Theorem 1, implies that the sequence $\left\{x_{n}\right\}$ is convergent, i.e. it exists $u \in L$ so that $\lim _{n \rightarrow \infty} x_{n}=u$. We will prove that $u$ is a fixed point of $S_{1}$. So,

$$
\begin{aligned}
\left\|u-S_{1} u, z\right\| \leq & \left\|u-x_{2 n+2}, z\right\|+\left\|x_{2 n+2}-S_{1} u, z\right\|=\left\|u-x_{n+1}, z\right\|+\left\|S_{2} x_{2 n+1}-S_{1} u, z\right\| \\
& \leq\left\|u-x_{2 n+2}, z\right\|+\sqrt{\alpha\left(\left\|u-S_{1} u, z\right\|^{2}+\left\|x_{2 n+1}-S_{2} x_{2 n+1}, z\right\|^{2}\right)+\beta\left\|u-x_{2 n+1}, z\right\|^{2}} \\
& =\left\|u-x_{2 n+2}, z\right\|+\sqrt{\alpha\left(\left\|u-S_{1} u, z\right\|^{2}+\left\|x_{2 n+1}-x_{2 n+2}, z\right\|^{2}\right)+\beta\left\|u-x_{n}, z\right\|^{2}},
\end{aligned}
$$

holds true for each $n \in \mathbf{N}$ and each $z \in L$. For $n \rightarrow \infty$, the latter is transformed as following $\|u-S u, z\| \leq \sqrt{\alpha}\|u-S u, z\|$, for each $z \in L$. Therefore, $S_{1} u=u$, i.e. $u$ is a fixed point of $S$. Analogously, $u$ is a fixed point of $S_{2}$, that is, $u$ is a common fixed point of $S_{1}$ and $S_{2}$. If suppose 
that $v$ is one other fixed point of $S_{2}$, and applying the inequality (17), we obtain the following $\|u-v, z\|^{2} \leq \beta\|u-v, z\|^{2}$. The latter implies that $u=v$.

Consequence 12. . Let $(L,\|, \cdot \cdot\|)$ be a 2-Banach space and $p, q \in \mathbf{N}$ be such that there exists mappings $S_{1}^{p}, S_{2}^{q}: L \rightarrow L$ and also exists $\alpha>0, \beta \geq 0,2 \alpha+\beta<1$ such that they satisfy the following

$$
\left\|S_{1}^{p} x-S_{2}^{q} y, z\right\|^{2} \leq \alpha\left(\left\|x-S_{2}^{q} y, z\right\|^{2}+\left\|y-S_{1}^{p} x, z\right\|^{2}\right)+\beta\|x-y, z\|^{2},
$$

for all $x, y, z \in L$. Then $S_{1}$ and $S_{2}$ have a unique common fixed point $u \in L$.

Proof. The proof is identical to the proof of Consequence 8 .

\section{Conclusion}

In this paper are proven several generalizations of known theorems of fixed point, and theorems for common fixed point of mapping to 2-Banach space. Given the importance of the fixed point theory and its application, logical question arises which other results can be generalized. For example, whether can be generalized the results given in [11].

\section{CONFLICT OF INTEREST}

No conflict of interest was declared by the authors.

\section{AUTHOR's CONTRIBUTIONS}

All authors contributed equally and significantly to writing this paper. All authors read and approved the final manuscript.

\section{REFERENCES}

[1] White, A., 2-Banach Spaces, Math. Nachr. Vol. 42 (1969), pp. 43-60

[2] Kir, M., Kiziltunc, H., Some New Fixed Point Theorems in 2-Normed Spaces, Int. Journal of Math. Analysis, Vol. 7 No. 58 (2013), pp. 2885-2890

[3] Chouhan, P., Malviya, N., Fixed Points of Expansive Type Mappings in 2-Banach Spaces, International Juornal of Analysis and Applications, Vol. 3 No. 1 (2013), pp. 60-67

[4] Hatikrishnan, P. K. , Ravindran, K. T., Some Properties of Accretive Operators in Linear 2Normed Spaces, International Mathematical Forum, Vol. 6 No. 59 (2011), 2941-2847

[5] Koparde, P. V., Waghmode, B. B., Kannan type mappings in Hilbert space, Scientist Phyl. Sciences, Vol. 3 No. 1 (1991), pp. 45-50

[6] Kannan, R., Some results on fixed points, Bull. Calc. Math. Soc. Vol. 60 No. 1, (1968), pp. 71-77

[7] Malčeski, R., Anevska, K., About the 2-Banach spaces, International Journal of Modern Engineering Research (IJMER), Vol. 4 Iss. 5 (2014), pp. 28-32

[8] Banach, S., Sur les operations dans les ensembles abstraits et leur application aux equations intégrales, Fund. Math. 2 (1922), pp. 133-181

[9] Gähler, S., Lineare 2-normierte Räume, Math. Nachr. 28 (1965), pp. 1-42

[10] Chatterjea, S. K., Fixed point theorems, C. R. Acad. Bulgare Sci., Vol. 25 No. 6 (1972), pp. $727-$ 730

[11] Malčeski, A., Malčeski, S., Anevska, K., Malčeski, R., New Extension of Kannan and Chatterjea Fixed Point Theorems on Complete Metric Spaces, British Journal of Mathematics \& Computer Science, Vol. 27, Iss. 1, pp. 1-10 